J. Product. \& Dev., 26(4): 865- 885 (2021)

\title{
QUINOA - YIELD AND ITS COMPONENT AS AFFECTED BY N FERTILIZATION, BIO-FERTILIZER AND MICRONUTRIENTS FERTILIZATION IN CALCAREOUS SOIL
}

\section{Zedan M. E.M., M.M. Sobh, M. Gouda and A.M.G. Ewis.}

Soil \&Water Dept. Faculty of Technology and Development, Zagazig Univ., Egypt.

* Corresponding author: e-mail address: mohamedzedan2468@gmail.com

\section{ABSTRACT}

A pot experiment was conducted at the Faculty of Technology and Development, Zagazig University, Sharkia Governorate, Egypt during 2018/2019 winter season to study the effect of integrated fertilization on the productivity of quinoa crop growing under calcareous soils. The experiment was laid out in split split plot arrangement using three replications. Main plots assigned to nitrogen fertilizers rates: $N_{0}, N_{1}, N_{2}, N_{3}$,

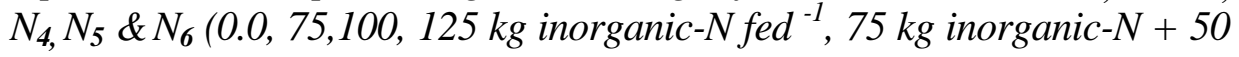
$\mathrm{kg}$ organic- $N, 100 \mathrm{~kg}$ inorganic- $N+25 \mathrm{~kg}$ organic- $N$ and $125 \mathrm{~kg}$ organic- $N$ $\left.\mathrm{fed}^{-1}\right)$. The sub plots were dedicated to bio-fertilizer rates of $b_{0}, b_{1}(0.0$ and $2 \mathrm{~L}$ fed-1). The sub-sub plots occupied to micronutrient rates of $T_{0}, T_{1}(0.0$ and $\left.2 \mathrm{~L} \mathrm{fed}^{-1}\right)$. The results indicated that the values of dry weight $(\mathrm{DW})$, grain yield $(G Y)$, straw yield $(S Y)$, biological yield $(B Y)$, harvest index $(H I)$, crude protein $(C P)$, nitrogen $(N)$, phosphorus $(P)$ and potassium $(K)$ uptakes by quinoa plant organs at flowering and harvest stage increased significantly with increasing bio-fertilizer rate, micronutrient rate and $N$ rate up to $100 \mathrm{fed}^{-1}$. Partial substitution of mineral nitrogen fertilizers $(M N F)$ by organic nitrogen fertilizer (ONF) had positive effect on all vegetative characters and chemical composition. Interaction between studied factors gave positive effect on most studied traits.

The best interaction treatment that achieved the highest values for most vegetative traits and chemical characters was $\left(N_{2} \times b_{1} \times T_{1}\right)$ or $\left(N_{4} \times\right.$ $\left.b_{1} \times T_{1}\right)$.

Keywords: Bio-fertilizer, N- fertilizer, chelate micronutrients fertilizers, Quinoa.

\section{INTRODUCTION}

Quinoa (Chenopodium quinoa Willd.) grown in a wide range of environments and characterized by its resistance to extreme environmental and climatic conditions. It can resist to drought stress (Eisa et al., 2012). It is tolerant to extreme soil salinity values, such as $52 \mathrm{mS} / \mathrm{cm}$.Quinoa requires temperate temperatures especially during flowering and for most genotypes short day-length to flower and produce seeds. Optimal mean temperatures are $10-18^{\circ} \mathrm{C}$ and it can 
resist both high $\left(30-32^{\circ} \mathrm{C}\right)$ and low temperature $\left(0-2^{\circ} \mathrm{C}\right)$, except during flowering (Garcia et al., 2015). While it is day-neutral in the other area (Oelke et. al.,1990). Therefore, quinoa fits to the Egyptian winter climate. Quinoa is now considered an alternative to traditional crops in a climate change conditions, considering its ability to adapt to marginal soils, droughts and frosts. Quinoa seeds are an exceptionally nutritious food source, owing to their high protein content with all essential amino acids, lack of gluten, and high content of several minerals, e.g., $\mathrm{Ca}, \mathrm{Mg}, \mathrm{Fe}$, and health-promoting (Karina et al.,2014). Quinoa has a good response to fertilization, in particular to nitrogen (Hakan 2015; Heba et al., 2019). Soil fertility is the major factor to determining the productivity of all planting systems; it is noted as a crucial problem facing agricultural development and crop output in soils especially. Phosphorus and nitrogen elements are considered the most important nutrients for root development, seed formation, growth and yield. (Beigzade et al., 2013) Potassium is one of the three Macro primary nutrients, which is necessary for plant growth (Rehm et al. 2002 and Lakudzala 2013). Micronutrients are as important as macronutrients in plant nutrition. The deficiency of micronutrients is considered one of the major causes of declining plant growth and yield of crops productivity (Taiwo et al., 2001 and Somani 2008). Plants growing in calcareous soils suffer from a lack of essential nutrients. Applying organic fertilizers have beneficial effects on soil microbial biomass and activity, enzymatic processes and status of nutrients in soil (Sarwar et. al.2010; Chukwu et al, 2012). On the other hand, bio-fertilizers are biological products containing live microbes which helps in enhancing the soil fertility either by fixing atmospheric nitrogen, solubilization of phosphorus, potassium mobilizing or decomposing organic wastes or by augmenting plant growth by producing growth hormones with their biological activities (Vessey 2003). Integrated soil fertility management is a holistic approach to enhance agricultural productivity and face problems related to poor soil fertility (Place et al., 2003).

Therefore, the objective of this work is to quantity the effect of integrated fertilization on yield of quinoa and its components in calcareous soil.

\section{MATERIALS AND METHODS}

A pot experiment was carried out at Faculty of Technology and Development, Zagazig University, Sharkia Governorate, Egypt during 2018/2019 winter season to investigate the effect of integrated fertilization on yield of quinoa and its components in calcareous soil. Main characteristics of the studied soil and compost using are recorded in Table 1. The compost was obtained from a factory in the Sharkia Governorate. Closed bottom plastic pots $(35 \mathrm{~cm}$ in diameter and 27 $\mathrm{cm}$ deep) filled with $20 \mathrm{~kg}$ air dried calcareous soil, which was transferred from 
Table 1. Some characteristic of soil and compost under study:

\begin{tabular}{|c|c|c|c|c|c|c|c|}
\hline \multicolumn{8}{|c|}{ Some physical properties of soil } \\
\hline $\begin{array}{c}\text { Sand } \\
\%\end{array}$ & $\begin{array}{c}\text { Silt } \\
\%\end{array}$ & $\begin{array}{c}\text { Clay } \\
\%\end{array}$ & $\begin{array}{c}\text { Textural } \\
\text { class }\end{array}$ & $\begin{array}{c}\text { Bulk densit } \\
\text { g/cm3 }\end{array}$ & \multicolumn{2}{|c|}{$\begin{array}{l}\text { Real density } \\
\text { g/cm3 }\end{array}$} & $\begin{array}{l}\text { S.P } \\
\%\end{array}$ \\
\hline 61 & 13 & 26 & $\begin{array}{c}\text { Sandy Clay } \\
\text { loam }\end{array}$ & 1.28 & \multicolumn{2}{|c|}{2.4} & 45 \\
\hline \multicolumn{8}{|c|}{ Some chemical properties of soil } \\
\hline \multirow{2}{*}{$\begin{array}{r}\mathbf{E C} \\
\left(\mathbf{d S m}^{-1}\right)\end{array}$} & \multirow[t]{2}{*}{ pH } & \multirow{2}{*}{$\begin{array}{c}\mathrm{CaCO}_{3} \\
\%\end{array}$} & \multirow[t]{2}{*}{$\mathrm{OM}\left(\mathrm{gKg}^{-1}\right)$} & \multirow{2}{*}{$\begin{array}{c}\text { Total N } \\
\left(\mathrm{gkg}^{-1}\right)\end{array}$} & \multicolumn{3}{|c|}{$\begin{array}{c}\text { Available } \\
\text { mgkg }^{-1}\end{array}$} \\
\hline & & & & & $\mathbf{N}$ & $\mathbf{P}$ & $\mathbf{K}$ \\
\hline 1.08 & 8.74 & 33.90 & 19.3 & 0.9 & 65 & 12 & 210 \\
\hline \multicolumn{8}{|c|}{ Chemical properties of compost } \\
\hline $\begin{array}{c}\mathrm{OM}_{\left(\mathrm{gkg}^{-1}\right)} \\
\end{array}$ & $\underset{\left(\mathrm{g} \mathrm{kg}^{-1}\right)}{\mathrm{OC}}$ & $\begin{array}{l}\mathrm{C} / \mathrm{N} \\
\text { ratio }\end{array}$ & $\begin{array}{c}\text { Total N } \\
\left(\mathrm{g} \mathrm{kg}^{-1}\right)\end{array}$ & $\begin{array}{l}\text { Total P } \\
\left(\mathrm{g} \mathrm{kg}^{-1}\right)\end{array}$ & \multicolumn{3}{|c|}{ Total K $\left(\mathrm{g} \mathrm{kg}^{-1}\right)$} \\
\hline 410 & 238 & $1: 17$ & 14 & 4.80 & \multicolumn{3}{|c|}{9.80} \\
\hline
\end{tabular}

the surface layer $(0-30 \mathrm{~cm})$ of Noubaria Research Station. The experiment was laid out in split split plot design arrangement with three replicates. The main plots were assigned to nitrogen fertilizer rates (mineral and organic), where $\mathrm{N}$ mineral fertilizer applied as ammonium sulphate (AS), $205 \mathrm{~g} \mathrm{~N} \mathrm{~kg}^{-1}$ while $\mathrm{N}$ organic fertilizer added as compost (C), $14 \mathrm{~g} \mathrm{~N} \mathrm{~kg}^{-1}$. The main plots treatments were as follows:

$\mathrm{N}_{0.0}=$ without $\mathrm{N}$ fertilizer

$\mathrm{N}_{\mathbf{1}}=75 \mathrm{~kg}$ inorganic- $\mathrm{N}$ fed ${ }^{-1}$

$\mathrm{N}_{2}=100 \mathrm{~kg}$ inorganic- $\mathrm{N}$ fed ${ }^{-1}$

$\mathrm{N}_{3}=125 \mathrm{~kg}$ inorganic- $\mathrm{N}$ fed ${ }^{-1}$

$$
\begin{aligned}
& \mathrm{N}_{4}=\mathrm{N}_{1}+50 \mathrm{~kg} \text { organic-N fed } \\
& \mathrm{N}_{5}=\mathrm{N}_{2}+25 \mathrm{~kg} \text { organic-N fed } \\
& \mathrm{N}_{6}=125 \mathrm{~kg} \text { organic- } \mathrm{N} \text { fed }
\end{aligned}
$$

The sub plots were dedicated to bio-fertilizer rates $\left(0.0\right.$ and $2 \mathrm{~L} \mathrm{fed}^{-1}$ i.e., $\mathrm{b}_{0}$, $\mathrm{b}_{1}$ ) of three microorganisms' species', nitrogen-fixing bacteria (Azotobacter Chroococum), phosphorus dissolving bacteria (Bacillus Megaterium Var.phosphaticum) and potassium solubilizing bacteria (Bacillus Circulans).

The sub-sub plots were occupied to micronutrient fertilizers in the form of chelated compounds for $\mathrm{Fe}, \mathrm{Cu}, \mathrm{Zn}$ and $\mathrm{Mn}$ at concentrations of 5, 2, 5 and $5 \%$, respectively (foliar sprayed at two levels 0.0 and $2 \mathrm{~L} \mathrm{fed}^{-1}$ i.e., $\mathrm{T}_{0}, \mathrm{~T}_{1}$ ). Both biofertilizers and chelate micronutrients were obtained from Egyptian Ministry of Agriculture (Central Labe of Organic Agricultural). On 6th/12/2018, twenty cleaned quinoa seeds (Chenopodium Quinoa Wild.) were sown in each pot with soil moisture at saturation by using tap water. For other irrigations, water was added when soil moisture had depleted to $75 \%$ of the total available water. Fifteen plants were left in each pot after thinning. The recommended rates of phosphorus 
(10.88 $\left.\mathrm{kg} \mathrm{P}^{\mathrm{P}} \mathrm{f}^{-1}\right)$ as Ca- superphosphate $\left(68 \mathrm{~g} \mathrm{P} \mathrm{kg}^{-1}\right)$, potassium $\left(20 \mathrm{~kg} \mathrm{~K} \mathrm{fed}^{-1}\right)$ as potassium sulphate (400 $\left.\mathrm{g} \mathrm{K} \mathrm{kg}^{-1}\right)$ as well as compost $\left(14 \mathrm{~g} \mathrm{~N} \mathrm{~kg}^{-1}\right)$ were added during soil preparation. Mineral nitrogen fertilizer was applied in three equal splitting doses; the first was after 21dayes from planting; the second after 25 days from the first and the third after 15 days from the second. Bio-fertilizers were added twice, the first with sowing and the second after 30 days from the first. Micronutrients fertilizers were added twice, the first after 70 days from sowing (panicle formation stage) and the second after 20 days from the first (full flowering stage). After 90 days from sowing five plants were taken from each pot. At harvest on 27/4/2019, all plants of each pot (ten plants) were taken and separated into grains and straw. The plant length (PL) cm, dry weight (DW), biological yield (BY), straw yield (SY) and grain yield (GY) were recorded (g $\left.\operatorname{pot}^{-1}\right)$. The plant samples were taken and oven dried at $70{ }^{\circ} \mathrm{C}$ until a constant weight then conserved for analysis. The N, P and K contents of the plants were determined by wet digestion using the standard methods as reported by Westerman (1990). Crude protein (CP) content was calculated by multiplying $\mathrm{N}$ content $\times 6.25$ according to Ronaled et al. (2005).

Harvest index $(\mathrm{HI})$ was calculated as a percent [(grain yield $\div$ total biological yield $) \times 100$ ]. Soil samples were taken before planting for analysis of some physical and chemical properties according to Sparks (1996).

\section{RESULTS AND DISCUSSION}

\section{1- Vegetative characters and yield quality:}

a) Effect of $N$ rate and source:

Data in Tables $(2,3)$ show that quinoa plant length (PL) at both flowering and harvesting stages increased significantly with increasing either mineral $\mathrm{N}$ fertilizer rate alone or in the case of partial replacement of it by organic $\mathrm{N}$ (compost), while the values decreased when the treatment $\mathrm{N}_{6}$ (compost, $125 \mathrm{~kg}$ $\mathrm{N}$ fed $^{-1}$ ) was added. In addition, data indicated that the values of quinoa dry weight (DW) at flowering stage, straw yield (SY), grain yield (GY) and biological yield (BY) at harvesting stage significantly affected by $\mathrm{N}$ fertilization treatments.

The above mentioned agronomic traits increased with increasing mineral $\mathrm{N}$ rate from zero up to $100 \mathrm{~kg} \mathrm{~N}$ fed $^{-1}$ then decreased at treatment $\mathrm{N}_{3}(125 \mathrm{~kg} \mathrm{~N}$ fed $\left.{ }^{1}\right)$ and then came back to increase again at treatment of $\mathrm{N}_{4}(75 \mathrm{~kg}$ inorganic- $\mathrm{N}+$ $50 \mathrm{~kg}$ organic-N). Generally using the treatment $\mathrm{N}_{5}(100 \mathrm{~kg}$ inorganic- $\mathrm{N}+25 \mathrm{~kg}$ organic-N) or $\mathrm{N}_{6}(125 \mathrm{~kg}$ organic-N) led to significant decreases in the values of (DW), (SY), (BY) and (GY) as compared to any N-treatment except $\mathrm{N}_{0}$ (control). The maximum values of DW (13.50), SY (62.61) and BY (99.30 $\mathrm{g} \mathrm{pot}^{-1}$ ) were recorded with $\mathrm{N}_{4}$ treatment, while for GY (36.92 and $36.69 \mathrm{~g} \mathrm{pot}^{-1}$ ) were reported 
Table2. Effect of nitrogen, bio and micronutrients fertilizers on vegetative characters as well as nutrient uptake by quinoa plant at flowering Stage.

\begin{tabular}{|c|c|c|c|c|c|}
\hline \multirow[t]{2}{*}{ Treatment } & Plant length & $\begin{array}{c}\text { Dry } \\
\text { weight }\end{array}$ & N-Uptake & $\begin{array}{c}\text { P- } \\
\text { Uptake }\end{array}$ & K-Uptake \\
\hline & cm & \multicolumn{4}{|c|}{ gram pot $^{-1}$} \\
\hline \multicolumn{6}{|c|}{$\mathrm{N}$-fertilizer rate $\left(\mathrm{kg} \mathrm{N} \mathrm{Fed}{ }^{-1}\right)$} \\
\hline $\mathbf{N}_{0}$ & 39.46 & 7.54 & 0.227 & 0.010 & 0.201 \\
\hline $\mathbf{N}_{1}$ & 48.25 & 12.11 & 0.440 & 0.017 & 0.353 \\
\hline $\mathbf{N}_{2}$ & 51.58 & 12.78 & 0.487 & 0.019 & 0.380 \\
\hline $\mathbf{N}_{3}$ & 54.33 & 11.24 & 0.432 & 0.016 & 0.334 \\
\hline $\mathbf{N}_{4}$ & 55.99 & 13.50 & 0.489 & 0.019 & 0.393 \\
\hline $\mathbf{N}_{5}$ & 58.21 & 11.06 & 0.410 & 0.015 & 0.321 \\
\hline $\mathbf{N}_{6}$ & 49.37 & 10.47 & 0.264 & 0.015 & 0.281 \\
\hline \multicolumn{6}{|c|}{ Bio. fertilizer rate $\left(\mathrm{L} \mathrm{Fed}{ }^{-1}\right)$} \\
\hline $\mathbf{b}_{0}$ & 49.36 & 10.71 & 0.377 & 0.015 & 0.303 \\
\hline $\mathbf{b}_{1}$ & 52.70 & 11.77 & 0.409 & 0.017 & 0.343 \\
\hline \multicolumn{6}{|c|}{ Micronutrients fertilizer rate $\left(\mathrm{L} \mathrm{Fed}^{-1}\right)$} \\
\hline $\mathbf{T}_{0}$ & 49.82 & 10.14 & 0.358 & 0.014 & 0.303 \\
\hline$T_{1}$ & 52.24 & 12.35 & 0.428 & 0.017 & 0.343 \\
\hline LSD 5\% a & 0.71 & 0.21 & 0.026 & 0.001 & 0.020 \\
\hline LSD 5\% b & 0.50 & 0.20 & 0.022 & 0.001 & 0.015 \\
\hline LSD 5\% c & 0.10 & 0.10 & 0.018 & 0.001 & 0.014 \\
\hline
\end{tabular}

Table3. Effect of nitrogen, bio and micronutrients fertilizers on quinoa yield and its quality at harvesting Stage.

\begin{tabular}{|c|c|c|c|c|c|c|}
\hline \multirow[t]{2}{*}{ Treatment } & $\begin{array}{c}\text { Plant } \\
\text { length }\end{array}$ & Straw yield & $\begin{array}{c}\text { Grain } \\
\text { yield }\end{array}$ & $\begin{array}{c}\text { Biological } \\
\text { yield }\end{array}$ & $\begin{array}{l}\text { Harves } \\
\text { t Index } \\
\end{array}$ & $\begin{array}{c}\text { Prote } \\
\text { in }\end{array}$ \\
\hline & $\mathbf{c m}$ & \multicolumn{3}{|c|}{$\operatorname{gram}_{\text {pot }}^{-1}$} & \multicolumn{2}{|c|}{$\%$} \\
\hline \multicolumn{7}{|c|}{$\mathrm{N}$-fertilizer rate $\left(\mathrm{kg} \mathrm{N} \mathrm{Fed}^{-1}\right)$} \\
\hline $\mathbf{N}_{\mathbf{0}}$ & 54.36 & 27.47 & 16.50 & 43.96 & 37.51 & 8.67 \\
\hline $\mathbf{N}_{1}$ & 74.05 & 54.62 & 34.35 & 88.97 & 38.55 & 14.62 \\
\hline $\mathbf{N}_{2}$ & 75.21 & 60.23 & 36.92 & 97.15 & 37.99 & 15.11 \\
\hline $\mathbf{N}_{3}$ & 79.30 & 55.83 & 33.54 & 89.37 & 37.53 & 13.20 \\
\hline $\mathbf{N}_{4}$ & 80.35 & 62.61 & 36.69 & 99.30 & 36.87 & 15.01 \\
\hline $\mathbf{N}_{5}$ & 83.22 & 47.28 & 31.67 & 78.95 & 40.14 & 13.18 \\
\hline $\mathbf{N}_{6}$ & 70.55 & 32.64 & 24.78 & 57.41 & 43.02 & 11.72 \\
\hline \multicolumn{7}{|c|}{ Bio. fertilizer rate $\left(\mathrm{L} \mathrm{Fed}^{-1}\right)$} \\
\hline $\mathbf{b}_{0}$ & 72.36 & 46.51 & 29.07 & 75.57 & 38.62 & 12.55 \\
\hline$b_{1}$ & 75.36 & 50.83 & 32.20 & 83.03 & 38.98 & 13.59 \\
\hline \multicolumn{7}{|c|}{ Micronutrients fertilizer rate $\left(\mathrm{L} \mathrm{Fed}^{-1}\right)$} \\
\hline $\mathbf{T}_{0}$ & 72.30 & 46.68 & 28.57 & 75.25 & 38.18 & 12.81 \\
\hline $\mathbf{T}_{1}$ & 75.42 & 50.66 & 32.70 & 83.35 & 39.42 & 13.34 \\
\hline LSD 5\%, a & 0.12 & 0.46 & 0.73 & 1.23 & 0.36 & 0.25 \\
\hline LSD 5\%,b & 0.14 & 0.43 & 0.71 & 0.96 & 0.30 & 0.18 \\
\hline LSD 5\%, c & 0.08 & 0.05 & 0.31 & 0.49 & 0.22 & 0.08 \\
\hline
\end{tabular}


with applying $\mathrm{N}_{2}\left(100 \mathrm{~kg} \mathrm{~N}\right.$ fed $\left.^{-1}\right)$ and $\mathrm{N}_{4}(75 \mathrm{~kg}$ inorganic- $\mathrm{N}+50 \mathrm{~kg}$ organic-N) respectively without significant different between them. These findings may be due to the integrated fertilization of chemical and organic fertilizers, which help in enhancing of nutrient status in soil especially that are low in organic matter (Umar Khan et. al., 2007). These results are in harmony with those obtained by Parra et al. (2019) and Heba et al. (2019). Concerning harvest index (HI), data recorded in Table 3 indicate that the values of this character showed a slightly effect by $\mathrm{N}$ fertilization rate. This finding attributed to the ratio of (increment value in grain yield / increment value in straw yield) at $\mathrm{N}_{6}$ (1.64) was higher than that the same ratio at $\mathrm{N}_{5}$ (1.15). Shafi et al. (2011) and Niguse and Kassaye (2018) reported that the harvest index has a positive relation with $\mathrm{N}$ fertilization rates as compared with control treatment $(0.0 \mathrm{~N}$ fertilizer rate) .The maximum value of 43.02 was obtained at $\mathrm{N}_{6}\left(125 \mathrm{~kg}\right.$ organic-N fed $\left.{ }^{-1}\right)$ followed by the value of 40.14 at $\mathrm{N}_{5}(100$ $\mathrm{kg}$ inorganic- $\mathrm{N}+25 \mathrm{~kg}$ organic- $\mathrm{N})$.

\section{b) Effect of bio-fertilizer}

As shown in Tables 2 and Table 3, using bio- fertilizer had a significant positive effect on (PL) and (DW) of quinoa at flowering stage and (PL), (SY), (GY), (BY) and (HI) at harvesting stage. As an average, using bio- fertilizer (2 $\left.\mathrm{L} \mathrm{fed}^{-1}\right)$ gave the best values of $\left(52.70 \mathrm{~cm}\right.$ and $\left.11.77 \mathrm{~g} \mathrm{pot}^{-1}\right)$ with increases of ( $6.77,9.90 \%$ ) for PL and DW at flowering stage while the values of (75.36 $\mathrm{cm}),\left(50.83 \mathrm{~g} \mathrm{pot}^{-1}\right),\left(32.20 \mathrm{~g} \mathrm{pot}^{-1}\right),\left(83.03 \mathrm{~g} \mathrm{pot}^{-1}\right)$ and $(39.98 \%)$ with increments of 4.15, , 9.29, 10.77, 9.87 and $0.93 \%$ for (PL), (SY), (GY), (BY) and $(\mathrm{HI})$ were realized at harvesting stage, respectively comparing to control treatment (zero bio- fertilizer). These results may be due the promoting effect of bacteria on plant growth and productivity by improving nitrogen fixation and raising phosphorus and potassium availability by releasing from its non soluble compounds in soil. Similar results are reported by Wali et al. (2018); Ewis (2020).

\section{c) Effect of micronutrients}

Data presented in Tables 2, 3 appeared that plant length, dry weight, grain, straw, biological yields and harvest index in the two studied growth stages increased significantly with foliar application of micronutrient fertilizer. Application of $2 \mathrm{~L} \mathrm{fed}^{-1}$ resulted in achieving maximum values of 52.24, cm, $12.35 \mathrm{~g} \mathrm{pot}^{-1}$ with increases of $4.86,21.79 \%$ for (PL) and (DW) at flowering stage, whereas the values of $75.42 \mathrm{~cm} 50.66 \mathrm{~g} \mathrm{pot}^{-1},, 32.70 \mathrm{~g} \mathrm{pot}^{-1}, 83.35 \mathrm{~g} \mathrm{pot}^{-1}$ and $39.42 \%$ with increments of, 4.32, $8.53,14.46,10.72$, and $3.25 \%$ for (PL), (SY), (GY), (BY) and (HI) were obtained at harvesting stage as compared to nonapplication micronutrient fertilizer $\left(\mathrm{T}_{0}\right)$, respectively. These results may be

attributed to the promoting effect of micronutrients on plant growth and 
productivity by positively affects enzymes, activation the cell physiology, improving photosynthetic activities. These results are in agreement with those obtained by Singh et al. (2017) and Stewart et al. (2021).

\section{d) Interaction effect:}

The statistical analysis of variance for data in (Tables 4, 4 continuous, 5 and 5 continuous) show different positive responses to interaction effect of mineral and organic $\mathrm{N}$ fertilizer rates, bio- and micronutrients fertilizer rates on quinoa yield and its components at flowering and harvesting stages. With respect to the effect of the interaction between $\mathrm{N}$ and bio-fertilizers rates, data reveal that the higher values of plant length at two studied periods were achieved at the treatment $\left(\mathrm{N}_{5} \times \mathrm{b}_{1}\right)$ with gained increments amounted to 50.80, 56.2 $0 \%$ while dry weight at flowering stage recorded the greater increase value of $86.90 \%$ by using the treatment $\left(\mathrm{N}_{4} \times \mathrm{b}_{1}\right)$ over the control treatments $\left(\mathrm{N}_{0}\right)$. On the other hand, higher values of $(\mathrm{SY}),(\mathrm{GY})$ and (BY) at harvest stage have been resulted from $\left(\mathrm{N}_{4} \times \mathrm{b}_{1}\right)$ with increases of 139.50, 131.50 and $136.70 \%$, respectively followed by the treatment of $\left(\mathrm{N}_{2} \times b_{1}\right)$ with increments of $130.50,127.00$ and $129.00 \%$ comparing to $\left(\mathrm{N}_{0}\right)$. As regards (HI), the treatments $\left(\mathrm{N}_{6} \times \mathrm{b}_{1}\right)$ or $\left(\mathrm{N}_{2} \times \mathrm{b}_{0}\right)$ gave the highest values.

Concerning the interaction effect of nitrogen fertilizer rates and micronutrient fertilizer rates, data presented in Tables 4, 4 continuous, 5 and 5 continuous indicate that treatment of $\left(\mathrm{N}_{5} \times \mathrm{T}_{1}\right)$ gave the highest values of plant length at flowering and harvest stages whereas, the interaction treatment $\left(\mathrm{N}_{6} \times \mathrm{T}_{1}\right)$ realized greatest harvest index value. In addition, the highest values of (DW), (SY), (GY), and (BY) were achieved as a result of $\left(\mathrm{N}_{4} \times \mathrm{T}_{1}\right)$ with increases of $99.90,140.00,154.00$ and $145.50 \%$, respectively as compared to $\left(\mathrm{N}_{0} \times \mathrm{T}_{0}\right)$.

Regarding the interaction effect of bio-fertilizer rates and micronutrient fertilizer rates, data emphasizes that, interaction of treatment $\left(b_{1} \times T_{1}\right)$ was the best one which recorded the maximum values for most studied vegetative characters with gained increments of 11.95, 33.00, 8.60, 18.00, 26.50 and $21.30 \%$ for (PL), (DW) at flowering stage, (PL), (SY), (GY) and (BY) at harvest stage over the control treatments $\left(\mathrm{b}_{0} \times \mathrm{T}_{0}\right)$. Also data indicate that the interaction between biofertilizer rates and micronutrient fertilizers had no significant effect on (HI) values.

In general, the results illustrated that the values of studied agronomic traits significantly affected by interaction between $\mathrm{N}$ levels, bio-fertilizer rates and micronutrient fertilizer rates. The best interaction treatment that achieved the highest values of 60.33 and $87.50 \mathrm{~cm}$ with increases of 57.40 and $66.90 \%$ for plant length at flowering and harvest stages, respectively was $\left(\mathrm{N}_{5} \times \mathrm{b}_{1} \times \mathrm{T}_{1}\right)$ as compared to $\left(\mathrm{N}_{0} \times \mathrm{b}_{0} \times \mathrm{T}_{0}\right)$ treatment. However, the highest values of dry weight at flowering stage, straw yield, grain yield and biological yield at harvest were recorded at $\left(\mathrm{N}_{4} \times \mathrm{b}_{1} \times \mathrm{T}_{1}\right)$ with increment of $123,168,179$ and $173 \%$ over 
Table4. Interaction effect of nitrogen, bio and micronutrients fertilizers on vegetative characters as well as nutrient uptalse by quinoa plant at flowering Stage.

\begin{tabular}{|c|c|c|c|c|c|c|c|}
\hline & Treatment & & $\begin{array}{l}\text { Plant } \\
\text { length }\end{array}$ & $\begin{array}{c}\text { Dry } \\
\text { weight }\end{array}$ & $\begin{array}{c}\text { N- } \\
\text { Uptalst }\end{array}$ & $\begin{array}{c}\text { P. } \\
\text { Uptalse }\end{array}$ & $\begin{array}{c}\mathbf{K}- \\
\text { Uptalse }\end{array}$ \\
\hline $\begin{array}{c}\text { N-Fert, rate } \\
\text { (lg Fed })\end{array}$ & $\begin{array}{l}\text { Bio, rate } \\
\text { (L Fed })\end{array}$ & $\begin{array}{l}\text { Mficro., rate } \\
\text { (L Fed })\end{array}$ & cm & \multicolumn{4}{|c|}{ gram por ${ }^{4}$} \\
\hline \multirow{6}{*}{ Ni } & \multirow{3}{*}{$b_{0}$} & $\mathrm{~T}_{0}$ & 38.33 & 7.08 & 0.192 & 0.009 & 0.187 \\
\hline & & $\mathrm{T}_{1}$ & 39.17 & 7.55 & 0.221 & 0.010 & 0.201 \\
\hline & & Mean & 38.75 & 7.31 & 0.206 & 0.010 & 0.194 \\
\hline & \multirow{3}{*}{$b_{i}$} & $\mathrm{~T}_{0}$ & 39.67 & 7.74 & 0.245 & 0.010 & 0.207 \\
\hline & & $\mathrm{T}_{1}$ & 40.67 & 7.79 & 0.251 & 0.010 & 0.208 \\
\hline & & Mean & 40.17 & 7.77 & 0.248 & 0.010 & 0.208 \\
\hline \multirow{6}{*}{$N_{i}$} & \multirow{3}{*}{$b_{0}$} & $\mathrm{~T}_{0}$ & 45.67 & 10.18 & 0.410 & 0.015 & 0.282 \\
\hline & & $\mathrm{T}_{1}$ & 46.33 & 13.19 & 0.488 & 0.020 & 0.402 \\
\hline & & Mean & 46.00 & 11.68 & 0.449 & 0.018 & 0.342 \\
\hline & \multirow{3}{*}{$\mathbf{b}_{i}$} & $\mathrm{~T}_{9}$ & 49.33 & 11.06 & 0.411 & 0.016 & 0.318 \\
\hline & & $\mathrm{T}_{1}$ & 51.67 & 14.02 & 0.453 & 0.018 & 0.412 \\
\hline & & Mean & 50.50 & 12.54 & 0.432 & 0.017 & 0.365 \\
\hline \multirow{6}{*}{$\mathrm{N}_{\mathrm{t}}$} & \multirow{3}{*}{$b_{0}$} & $\mathrm{~T}_{2}$ & 47.67 & 11.15 & 0.443 & 0.018 & 0.351 \\
\hline & & $\mathrm{T}_{1}$ & 48.67 & 13.63 & 0.517 & 0.018 & 0.371 \\
\hline & & Mean & 48.17 & 12.39 & 0.480 & 0.018 & 0.361 \\
\hline & \multirow{3}{*}{$\mathbf{b}_{i}$} & $\mathrm{~T}_{0}$ & 55.67 & 11.86 & 0.450 & 0.016 & 0.349 \\
\hline & & $\mathrm{T}_{1}$ & 54.33 & 14.47 & 0.539 & 0.024 & 0.450 \\
\hline & & Mean & 55.00 & 13.17 & 0.495 & 0.020 & 0.399 \\
\hline \multirow{6}{*}{ Nis } & \multirow{3}{*}{ bo } & $\mathrm{T}_{9}$ & 51.00 & 10.16 & 0.384 & 0.014 & 0.313 \\
\hline & & $T_{1}$ & 53.67 & 10.40 & 0.398 & 0.014 & 0.299 \\
\hline & & Mean & 52.34 & 10.28 & 0.391 & 0.014 & 0.306 \\
\hline & \multirow{3}{*}{$b_{i}$} & $\mathrm{~T}_{0}$ & 55.33 & 10.35 & 0.387 & 0.014 & 0.320 \\
\hline & & $\mathrm{T}_{1}$ & 57.33 & 14.06 & 0.560 & 0.021 & 0.402 \\
\hline & & Mean & 56.33 & 12.20 & 0.474 & 0.018 & 0.361 \\
\hline \multirow{6}{*}{$\mathrm{N}_{4}$} & \multirow{3}{*}{$b_{0}$} & $\mathrm{~T}_{0}$ & 52.87 & 11.99 & 0.451 & 0.017 & 0.363 \\
\hline & & $\mathrm{T}_{1}$ & 56.33 & 13.82 & 0.492 & 0.019 & 0.341 \\
\hline & & Mean & 54.60 & 12.91 & 0.472 & 0.018 & 0.352 \\
\hline & \multirow{3}{*}{$b_{i}$} & $\mathrm{~T}_{9}$ & 54.93 & 12.39 & 0.451 & 0.021 & 0.427 \\
\hline & & $\mathrm{T}_{1}$ & 59.83 & 15.80 & 0.564 & 0.021 & 0.440 \\
\hline & & Mean & 57.38 & 14.09 & 0.507 & 0.021 & 0.433 \\
\hline \multirow{6}{*}{ Ns } & \multirow{3}{*}{$b_{0}$} & $\mathrm{~T}_{0}$ & 56.67 & 10.06 & 0.369 & 0.013 & 0.314 \\
\hline & & $\mathrm{T}_{1}$ & 57.17 & 11.12 & 0.404 & 0.013 & 0.291 \\
\hline & & Mean & 56.92 & 10.59 & 0.386 & 0.013 & 0.303 \\
\hline & \multirow{3}{*}{$b_{i}$} & $\mathrm{~T}_{0}$ & 58.67 & 9.83 & 0.343 & 0.013 & 0.303 \\
\hline & & $\mathrm{T}_{1}$ & 60.33 & 13.24 & 0.523 & 0.018 & 0.374 \\
\hline & & Mean & 59.50 & 11.54 & 0.433 & 0.016 & 0.339 \\
\hline \multirow{6}{*}{$N_{6}$} & \multirow{3}{*}{$b_{0}$} & $\mathrm{~T}_{9}$ & 45.33 & 8.49 & 0.239 & 0.012 & 0.241 \\
\hline & & $T_{1}$ & 52.13 & 11.18 & 0.273 & 0.017 & 0.291 \\
\hline & & Mean & 48.73 & 9.84 & 0.256 & 0.014 & 0.266 \\
\hline & \multirow{3}{*}{$\mathbf{b}_{i}$} & $\mathrm{~T}_{0}$ & 46.33 & 9.65 & 0.229 & 0.013 & 0.264 \\
\hline & & $\mathrm{T}_{1}$ & 53.67 & 12.58 & 0.314 & 0.017 & 0.326 \\
\hline & & Mean & 50.00 & 11.11 & 0.272 & 0.015 & 0.295 \\
\hline
\end{tabular}


Continuous in Tables.

\begin{tabular}{|c|c|c|c|c|c|c|c|}
\hline \multicolumn{3}{|c|}{ Treatment } & $\begin{array}{l}\text { Plant } \\
\text { length }\end{array}$ & $\begin{array}{l}\text { Dry } \\
\text { weight }\end{array}$ & $\begin{array}{c}\text { N- } \\
\text { Uptaliee }\end{array}$ & $\begin{array}{c}\text { P- } \\
\text { Uptake }\end{array}$ & $\begin{array}{c}\text { K- } \\
\text { Uptalize }\end{array}$ \\
\hline $\begin{array}{c}\text { N-Fert, } \\
\text { rate } \\
\left(\text { (kg Fed }^{-1}\right)\end{array}$ & \multicolumn{2}{|c|}{$\begin{array}{l}\text { Micro, rate } \\
\left(\mathrm{L} \text { Fed }{ }^{-1}\right)\end{array}$} & $\mathrm{cm}$ & \multicolumn{4}{|c|}{ gram pot ${ }^{-1}$} \\
\hline \multirow{2}{*}{$\mathrm{N}_{0}$} & & $\mathrm{~T}_{0}$ & 39.00 & 7.41 & 0.219 & 0.010 & 0.197 \\
\hline & & $\mathrm{T}_{1}$ & 39.92 & 7.67 & 0.236 & 0.010 & 0.205 \\
\hline \multirow{2}{*}{$\mathrm{N}_{1}$} & & $\mathrm{~T}_{\mathrm{u}}$ & 4750 & 10.62 & 0.410 & 0.015 & 0.300 \\
\hline & & $\mathrm{T}_{1}$ & 49.00 & 13.61 & 0.471 & 0.019 & 0.407 \\
\hline \multirow{2}{*}{$\mathrm{N}_{2}$} & & $\mathrm{~T}_{0}$ & 51.67 & 11.51 & 0.446 & 0.017 & 0.350 \\
\hline & & $\mathrm{T}_{1}$ & 51.50 & 14.05 & 0.528 & 0.021 & 0.410 \\
\hline \multirow{2}{*}{$\mathrm{N}_{2}$} & & $\mathrm{~T}_{0}$ & 53.17 & 10.25 & 0.386 & 0.014 & 0.316 \\
\hline & & $\mathrm{T}_{1}$ & 5550 & 12.23 & 0.479 & 0.017 & 0.351 \\
\hline \multirow{2}{*}{$\mathrm{N}_{4}$} & & $\mathrm{~T}_{0}$ & 5390 & 12.19 & 0.451 & 0.019 & 0.395 \\
\hline & & $\mathrm{T}_{1}$ & 58.08 & 14.81 & 0.528 & 0.020 & 0.390 \\
\hline \multirow{2}{*}{$\mathrm{N}_{s}$} & & $\mathrm{~T}_{0}$ & 57.67 & 9.94 & 0.356 & 0.013 & 0.309 \\
\hline & & $\mathrm{T}_{1}$ & 58.75 & 12.18 & 0.463 & 0.016 & 0.333 \\
\hline \multirow{2}{*}{$\mathrm{N}_{6}$} & & $\mathrm{~T}_{0}$ & 45.83 & 9.07 & 0.234 & 0.012 & 0.253 \\
\hline & & $\mathrm{T}_{1}$ & 5290 & 11.88 & 0.294 & 0.017 & 0.309 \\
\hline \multirow{4}{*}{$\begin{array}{l}\text { Bio. Fert, } \\
\text { rate } \\
\text { (L Fed }^{-1} \text { ) }\end{array}$} & \multirow{2}{*}{$\mathrm{b}_{0}$} & $\mathrm{~T}_{0}$ & 48.22 & 9.87 & 0.355 & 0.014 & 0.293 \\
\hline & & $\mathrm{T}_{1}$ & 50.50 & 11.56 & 0.399 & 0.016 & 0.314 \\
\hline & \multirow{2}{*}{$\mathrm{b}_{1}$} & $\mathrm{~T}_{0}$ & 51.42 & 10.41 & 0.360 & 0.015 & 0.313 \\
\hline & & $\mathrm{T}_{1}$ & 5398 & 13.14 & 0.458 & 0.018 & 0.373 \\
\hline \multicolumn{3}{|c|}{$\operatorname{LSD}_{\Omega} \quad a * b$} & 0.25 & 0.25 & NS & NS & NS \\
\hline \multicolumn{3}{|c|}{$\operatorname{LSD}_{\mathrm{N}} \quad \mathrm{a}^{*} \mathrm{c}$} & 0.25 & 0.25 & NS & 0.001 & 0.036 \\
\hline \multicolumn{3}{|c|}{$\operatorname{LSD}_{5 \mathrm{~s}} \quad \mathrm{~b}^{\star} \mathrm{c}$} & 0.13 & 0.14 & 0.025 & 0.001 & 0.019 \\
\hline \multicolumn{3}{|c|}{$\operatorname{LSD}_{m} \mathrm{a}^{*} \mathrm{~b}^{*} \mathrm{c}$} & 0.35 & 0.36 & 0.067 & 0.002 & 0.051 \\
\hline
\end{tabular}


Table 5. Interaction effect of nitrogen, bio and micronutrients fertilizers on quinoa yield and its quality at harvesting Stage.

\begin{tabular}{|c|c|c|c|c|c|c|c|c|}
\hline \multicolumn{3}{|c|}{ Treatmeat } & $\begin{array}{l}\text { Plant } \\
\text { lengeth }\end{array}$ & $\begin{array}{c}\text { Strnir } \\
\text { rield }\end{array}$ & $\begin{array}{l}\text { Grain } \\
\text { rield }\end{array}$ & $\begin{array}{c}\text { Biological } \\
\text { rield }\end{array}$ & $\begin{array}{l}\text { Harvest } \\
\text { Inder }\end{array}$ & Proteie \\
\hline $\begin{array}{l}\text { N.Fert, } \\
\text { ratte } \\
\left(\mathrm{kgg}^{2} \text { Fed }\right)^{2}\end{array}$ & $\begin{array}{l}\text { Bio., rate } \\
\text { (L Fed ') }\end{array}$ & $\begin{array}{l}\text { Micro, } \\
\text { rate } \\
(L \text { Fed'1) }\end{array}$ & $\mathrm{cm}$ & \multicolumn{3}{|c|}{ gram pot ${ }^{4}$} & \multicolumn{2}{|c|}{$\%$} \\
\hline \multirow{6}{*}{$\mathrm{N}$} & \multirow{3}{*}{$b_{a}$} & $\mathrm{~T}_{6}$ & 52.44 & 25.78 & 15.07 & 40.85 & 36.89 & 7.96 \\
\hline & & $\mathrm{I}_{1}$ & 53.67 & 26.75 & 16.25 & 43.00 & 37.79 & 8.32 \\
\hline & & Mean & 53.05 & 26.26 & 15.66 & 41.92 & 37.34 & 8.14 \\
\hline & \multirow{3}{*}{$b_{t}$} & $\mathrm{~T}_{6}$ & 53.33 & 28.01 & 16.82 & 44.83 & 37.52 & 8.78 \\
\hline & & $\mathrm{T}_{1}$ & 58.00 & 29.34 & 17.85 & 47.19 & 37.82 & 9.63 \\
\hline & & Iean & 55.67 & 28.68 & 17.34 & 46.01 & 37.67 & 9.20 \\
\hline \multirow{6}{*}{$\mathrm{N}_{\mathrm{i}}$} & \multirow{3}{*}{$b_{0}$} & $\mathrm{~T}_{6}$ & 71.67 & 50.84 & 31.51 & 82.35 & 38.26 & 13.27 \\
\hline & & $\mathrm{I}_{1}$ & 73.67 & 57.67 & 32.94 & 90.61 & 3635 & 13.81 \\
\hline & & Mean & 72.67 & 54.25 & 32.23 & 86.48 & 37.31 & 13.54 \\
\hline & \multirow{3}{*}{$b_{t}$} & $\mathrm{~T}_{0}$ & 74.33 & 52.01 & 32.63 & 84.64 & 38.55 & 15.62 \\
\hline & & $\mathrm{T}_{1}$ & 76.52 & 57.95 & 40.33 & 98.28 & 41.03 & 15.76 \\
\hline & & Mean & 75.43 & 54.98 & 36.48 & 91.46 & 39.79 & 15.69 \\
\hline \multirow{6}{*}{$\mathrm{N}_{2}$} & \multirow{3}{*}{$b_{0}$} & $\mathrm{~T}_{\mathrm{b}}$ & 72.33 & 55.77 & 33.97 & 89.74 & 37.85 & 14.53 \\
\hline & & $\mathrm{T}_{1}$ & 74.52 & 58.52 & 38.81 & 97.33 & 39.87 & 14.25 \\
\hline & & Iean & 73.43 & 57.15 & 36.39 & 93.54 & 38.96 & 14.39 \\
\hline & \multirow{3}{*}{$b_{1}$} & $\mathrm{~T}_{6}$ & 75.33 & 57.88 & 33.64 & 91.52 & 36.75 & 15.75 \\
\hline & & $\mathrm{I}_{1}$ & 78.67 & 68.76 & 41.26 & 110.02 & 37.50 & 15.92 \\
\hline & & Mean & 77.00 & 63.32 & 37.45 & 100.77 & 37.12 & 15.84 \\
\hline \multirow{6}{*}{$\mathrm{N}$} & \multirow{3}{*}{ bo } & $\mathrm{T}_{6}$ & 76.52 & 51.53 & 30.92 & 82.45 & 37.49 & 12.27 \\
\hline & & $\mathrm{T}_{1}$ & 79.33 & 55.76 & 33.62 & 89.38 & 37.61 & 13.54 \\
\hline & & Mean & 77.93 & 53.64 & 32.27 & 85.92 & 37.55 & 12.90 \\
\hline & \multirow{3}{*}{$b_{t}$} & $\mathrm{~T}_{\mathrm{b}}$ & 79.67 & 55.29 & 33.12 & 88.41 & 37.46 & 12.96 \\
\hline & & $\mathrm{T}_{1}$ & 81.67 & 60.73 & 36.52 & 97.25 & 37.55 & 14.02 \\
\hline & & Iean & 80.67 & 58.01 & 34.82 & 92.83 & 3750 & 13.49 \\
\hline \multirow{6}{*}{$\mathrm{N}_{4}$} & \multirow{3}{*}{$b_{0}$} & $\mathrm{~T}_{6}$ & 77.48 & 58.66 & 31.53 & 90.19 & 3496 & 14.38 \\
\hline & & $\mathrm{T}_{1}$ & 80.15 & 60.01 & 38.87 & 98.88 & 3931 & 15.08 \\
\hline & & Mean & 78.82 & 59.34 & 35.20 & 94.54 & 37.13 & 14.73 \\
\hline & \multirow{3}{*}{$b_{t}$} & $\mathrm{~T}_{6}$ & 80.45 & 62.48 & 34.26 & 96.74 & 35.41 & 15.14 \\
\hline & & $\mathrm{I}_{1}$ & 83.33 & 69.28 & 42.11 & 111.39 & 37.79 & 15.42 \\
\hline & & Мean & 81.89 & 65.88 & 38.19 & 104.07 & 36.60 & 15.28 \\
\hline \multirow{6}{*}{ Ns } & \multirow{3}{*}{$b_{0}$} & $\mathrm{~T}_{\mathrm{f}}$ & 78.86 & 46.87 & 29.19 & 76.06 & 38.37 & 12.54 \\
\hline & & $\mathrm{T}_{1}$ & 84.17 & 44.87 & 31.70 & 76.57 & 41.39 & 12.79 \\
\hline & & Iean & 81.51 & 45.87 & 30.45 & 76.31 & 39.88 & 12.67 \\
\hline & \multirow{3}{*}{$b_{1}$} & $\mathrm{~T}_{6}$ & 82.33 & 43.84 & 30.97 & 74.81 & 41.39 & 13.66 \\
\hline & & $\mathrm{T}_{1}$ & 87.50 & 53.53 & 34.82 & 88.35 & 39.41 & 13.74 \\
\hline & & Mean & 84.92 & 48.68 & 32.90 & 81.58 & 40.40 & 13.70 \\
\hline \multirow{6}{*}{$\mathrm{N}_{\mathrm{s}}$} & \multirow{3}{*}{ bo } & $\mathrm{T}_{6}$ & 67.60 & 28.41 & 20.25 & 48.66 & 41.61 & 10.19 \\
\hline & & $\mathrm{T}_{1}$ & 70.67 & 29.67 & 22.33 & 52.00 & 4295 & 12.77 \\
\hline & & Mean & 69.13 & 29.04 & 21.29 & 50.33 & 42.28 & 11.48 \\
\hline & & $\mathrm{T}_{\mathrm{b}}$ & 69.92 & 36.12 & 26.14 & 62.26 & 41.98 & 12.25 \\
\hline & $b_{t}$ & $\mathrm{T1}$ & 74.00 & 36.34 & 30.39 & 66.73 & 45.54 & 11.67 \\
\hline & & Mean & 71.96 & 36.23 & 28.27 & 64.50 & 43.76 & 11.96 \\
\hline
\end{tabular}




\section{Continuous in Table 5.}

\begin{tabular}{|c|c|c|c|c|c|c|c|c|}
\hline \multicolumn{3}{|c|}{ Trestment } & $\begin{array}{l}\text { Plant } \\
\text { length }\end{array}$ & $\begin{array}{l}\text { Straut } \\
\text { yield }\end{array}$ & $\begin{array}{c}\text { Grain } \\
\text { jield }\end{array}$ & $\begin{array}{c}\text { Biological } \\
\text { yield }\end{array}$ & $\begin{array}{l}\text { Harvest } \\
\text { Index }\end{array}$ & Protein \\
\hline $\begin{array}{c}\text { X-Ferth } \\
\text { rate } \\
\left(\mathrm{kg} \mathrm{Fed} \mathrm{d}^{3}\right)\end{array}$ & \multicolumn{2}{|c|}{$\begin{array}{l}\text { Micro, rate } \\
\left.(\mathrm{LFed})^{3}\right)\end{array}$} & $\mathrm{cm}$ & \multicolumn{3}{|c|}{$\operatorname{gram}_{\mathrm{pot}}{ }^{-1}$} & \multicolumn{2}{|c|}{$9 \%$} \\
\hline \multirow{2}{*}{$\mathrm{N}_{0}$} & \multicolumn{2}{|c|}{$\mathrm{T}_{0}$} & 5239 & 26.99 & 15.94 & 4283 & 37.21 & 837 \\
\hline & \multicolumn{2}{|c|}{$\mathbf{T}_{1}$} & 55.83 & 28.04 & 17.05 & 45.09 & 37.81 & 898 \\
\hline \multirow{2}{*}{$\mathrm{N}_{1}$} & \multicolumn{2}{|c|}{$\mathrm{T}_{0}$} & 73.00 & 51.42 & 3207 & 83.49 & 38.41 & 14.45 \\
\hline & \multicolumn{2}{|c|}{$\mathrm{T}_{1}$} & 75.09 & 57.81 & 36.63 & 94.44 & 38.69 & 14.79 \\
\hline \multirow{2}{*}{$\mathrm{N}_{2}$} & \multicolumn{2}{|c|}{$\mathrm{T}_{0}$} & 73.83 & 56.83 & 33.81 & 90.64 & 37.30 & 15.14 \\
\hline & \multicolumn{2}{|c|}{$\mathrm{T}_{1}$} & 76.59 & 63.64 & 40.03 & 103.67 & 38.68 & 15.08 \\
\hline \multirow{2}{*}{$\mathrm{N}}$, & \multicolumn{2}{|c|}{$\mathrm{T}_{0}$} & 78.09 & 53.41 & 3202 & 85.43 & 37.47 & 12.62 \\
\hline & \multicolumn{2}{|c|}{$\mathrm{T}_{1}$} & 80.50 & 58.24 & 35.07 & 93.31 & 37.98 & 13.78 \\
\hline \multirow{2}{*}{$\mathrm{N}_{4}$} & \multicolumn{2}{|c|}{$\mathrm{T}_{0}$} & 78.97 & 60.57 & 32.90 & 93.47 & 35.18 & 14.76 \\
\hline & \multicolumn{2}{|c|}{$\mathbf{T}_{1}$} & 81.74 & 64.65 & 40.49 & 105.14 & 38.55 & 15.25 \\
\hline \multirow{2}{*}{$\mathrm{N}_{2}$} & \multicolumn{2}{|c|}{$\mathrm{T}_{0}$} & 80.60 & 45.35 & 30.08 & 75.43 & 39.99 & 13.10 \\
\hline & \multicolumn{2}{|c|}{$\mathbf{T}_{1}$} & 85.83 & 49.20 & 33.26 & $\$ 246$ & 40.40 & 13.27 \\
\hline \multirow{2}{*}{$\mathrm{N}_{0}$} & \multicolumn{2}{|c|}{$\mathrm{T}_{0}$} & 68.76 & 3226 & 23.19 & 58.45 & 41.50 & 11.22 \\
\hline & \multicolumn{2}{|c|}{$\mathrm{T}_{1}$} & 72.33 & 33.01 & 2636 & 59.37 & 44.24 & $12 n$ \\
\hline \multirow{4}{*}{$\begin{array}{l}\text { Bio. Ferth- } \\
\text { rate } \\
\left.\text { (L Fed }{ }^{-3}\right)\end{array}$} & \multirow{2}{*}{$b_{6}$} & $\mathrm{~T}_{0}$ & 70.99 & 45.41 & 27.49 & 7290 & 37.91 & 12.16 \\
\hline & & $\mathbf{T}_{1}$ & 73.74 & 47.61 & 30.65 & 78.26 & 39.33 & 1294 \\
\hline & \multirow{2}{*}{$b_{1}$} & $\mathrm{~T}_{0}$ & 73.62 & 4795 & 29.65 & 77.60 & 38.44 & 13.45 \\
\hline & & $\mathrm{T}_{1}$ & 77.10 & 53.70 & 34.75 & 88.46 & 39.52 & 13.74 \\
\hline \multicolumn{3}{|c|}{$\operatorname{LSD}_{n a} \quad \mathrm{a}^{*} \mathrm{~b}$} & 0.22 & 0.13 & 0.83 & 1.30 & 0.58 & 0.22 \\
\hline \multicolumn{3}{|c|}{$\operatorname{LSD}_{x: \mathrm{a}} \quad \mathrm{a}^{*} \mathrm{c}$} & 0.22 & 0.13 & 0.83 & 1.30 & 0.58 & 0.22 \\
\hline \multicolumn{3}{|c|}{$\operatorname{LSD}_{n: 4} \quad b^{*} c$} & 0.12 & 0.07 & 0.44 & 0.70 & NS & 0.12 \\
\hline \multicolumn{3}{|c|}{ LSD $_{n: \Delta} a^{*} b^{*} c$} & 0.31 & 0.18 & 1.18 & 1.84 & 0.62 & 0.32 \\
\hline
\end{tabular}

$\left(\mathrm{N}_{0} \times \mathrm{b}_{0} \times \mathrm{T}_{0}\right)$, respectively. Additionally, data appeared that there is no significant difference between the interaction treatments of $\left(\mathrm{N}_{4} \times \mathrm{b}_{1} \times \mathrm{T}_{1}\right)$ and $\left(\mathrm{N}_{2}\right.$ $\left.\times b_{1} \times T_{1}\right)$. Notwithstanding, treatments of $\left(\mathrm{N}_{4} \times \mathrm{b}_{1} \times \mathrm{T}_{1}\right)$ was superior to $\left(\mathrm{N}_{2} \times \mathrm{b}_{1}\right.$ $\left.\times \mathrm{T}_{1}\right)$. This means that, we can save about $25 \mathrm{~kg}$ of mineral-N fed ${ }^{-1}$ and replace it by adding $50 \mathrm{~kg}$ of organic- $\mathrm{N} \mathrm{fed}{ }^{-1}$ as compost. These association positive for the 
studied agronomic traits could be attributed to the beneficial effect of integrate organic, inorganic and bio -fertilizers to improve nutrient status in soil. These results coincide with those obtained by Ashik et al. (2016) and Bilal et al. (2017).

\section{2- Chemical composition and nutrients uptake}

a) Effect of $N$ fertilization rates (mineral and organic)

Data in Tables 2, 3 and 6, indicate that $\mathrm{N}, \mathrm{P}, \mathrm{K}$-uptake $\left(\mathrm{g} \mathrm{pot}^{-1}\right)$ by quinoa plant organs as well as total nutrient uptake (sum of element uptake by straw and grains) at flowering and harvest stages and grains protein content (\%), increased significantly with increasing mineral $\mathrm{N}$ fertilizer rate up to $100 \mathrm{~kg} \mathrm{~N}$ $\mathrm{fed}^{-1}$. In addition, data show that partial substitution of mineral nitrogen fertilizers (MNF) by organic $\mathrm{N}$ fertilizer (ONF) i.e., $75 \mathrm{~kg}$ mineral- $\mathrm{N}+50 \mathrm{~kg}$ organic- $\mathrm{N} \mathrm{fed}{ }^{-1}$ led to significant increases of nutrient uptake. This result might be due to the crucial function of nitrogen to stimulating metabolic activities, accelerating metabolic products and hence improving growth leading to a better yield qualitatively as well as quantitatively as already discussed (results in Tables $2 \& 3$ ). These results are in conformity with those obtained by Hakan (2015); and Abdulrahman et al. (2019). On the other hand, data also clearly appear that the values of abovementioned parameters markedly decreased with added $100 \mathrm{~kg}$ inorganic- $\mathrm{N}+25 \mathrm{~kg}$ organic- $\mathrm{N}$ fed ${ }^{-1}$ and severely decreased with applying $125 \mathrm{~kg}$ organic- $\mathrm{N}$ fed ${ }^{-1}$ (Tables $3 \& 6$ ). In general, the relative decreases of grains protein content, total $\mathrm{N}, \mathrm{P}$ and $\mathrm{K}$-uptake at harvest stage due to applying (100 kg inorganic- $\mathrm{N}+25 \mathrm{~kg}^{-}$organic- $\mathrm{N}$ fed $^{-1}$ ) as compared to applying (100 kg mineral- $\mathrm{N} \mathrm{fed}^{-1}$ ) were 12.80, 29.40, 26.50 and $30.00 \%$, respectively. In contrast, the relative decreases of the same parameters were $22.40,61.90,30.60$ and $53.00 \%$ as a result of applying (125 kg organic-N fed

$\left.{ }^{1}\right)$, respectively. This depressing effect possibly could be explained by the wide $\mathrm{C}: \mathrm{N}$ ratio of compost which decreased the availability of $\mathrm{N}$ required for growing plants through its immobilization into organic $\mathrm{N}$ forms. Nitrogen, phosphorus and potassium-uptake by quinoa plants at flowering stage as well as by straw and grains at harvest stage took the same trend as for total nutrient uptake with application of $\mathrm{N}$ fertilization rates.

\section{b) Effect of bio-fertilizer rate:}

Data in Tables 2, 3 and 6 explicitly indicate that bio- fertilizer rate had a significant effect of $\mathrm{N}, \mathrm{P}, \mathrm{K}$-uptake $\left(\mathrm{g} \mathrm{pot}^{-1}\right)$ by quinoa plant organs as well as total nutrient uptake at flowering and harvest stages and crude protein content (\%). As an average, the increments were $8.50,13.30$ and $13.2 \%$ for $\mathrm{N}, \mathrm{P}$ and $\mathrm{K}$ uptake by quinoa plant at flowering stage respectively. While the gained increases for grains protein content, $\mathrm{N}, \mathrm{P}$ and $\mathrm{K}$-uptake by straw and grains as well as total nutrient uptake at maturity stage were 8.30, (6.30, $4.20 \& 9.40 \%)$ straw, ( 19.50, 


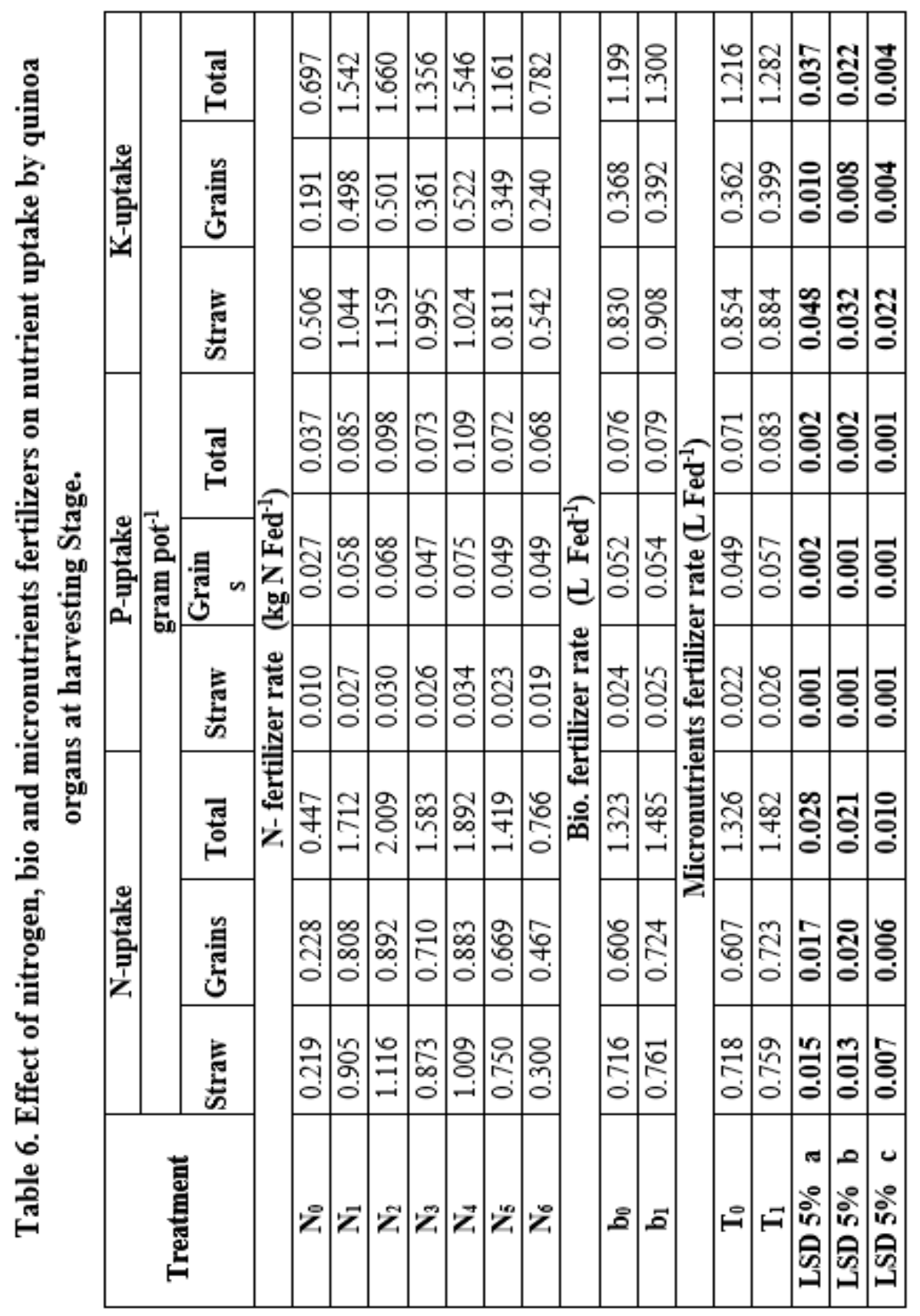


$3.80 \& 6.50 \%)$ grains and $(12.20,3.90$ and $8.40 \%)$ total, respectively as compared to non-application of bio-fertilizer. These findings can possibly due to the stimulation effect of bio-fertilizers in increasing the biological nitrogen fixation (BNF), solubilization of nutrients and increased its availability or through increased hormonal action and antibiosis. These results coincide with mentioned by Ewis (2019) and Ewis (2020) whom reported that application of bio- fertilizers recorded the highest available and uptake of NPK and significantly superior over control treatment.

\section{c) Effect of micronutrient fertilizer rate:}

Data clearly demonstrate that chemical composition, crude protein content and nutrient uptake by quinoa plant organs at flowering and maturity stages significantly influenced by foliar spraying of micronutrients (Tables 2, 3\&6). Using micronutrients solution at a level of $2 \mathrm{Lfed}^{-1}$ gave maximum values of $0.428,0.017,0.343,1.482,0.083$ and $1.282 \mathrm{~g} \mathrm{pot}^{-1}$ and $13.34 \%$ with increments of $19.60,21.40,13.20,11.76,16.90,5.40$ and $4.10 \%$ for total $\mathrm{N}, \mathrm{P}, \mathrm{K}$-uptake at flowering and harvest stages and quinoa protein content, respectively as compared to non-fertilized treatment. Furthermore, data indicate that N, P, K-uptake at flowering and harvest stages by quinoa organs took the same trend as for total nutrient uptake with applying of micronutrients fertilizer. Bender et al. (2013) and Stewart et al. (2021) obtained similar results.

\section{d) Interaction effect:}

Data illustrated in Tables $4 \& 7$ and continuous show that the interaction between $\mathrm{N}$ levels and bio-fertilizer rates had no significant effect on values of $\mathrm{N}$, $\mathrm{P}$ and $\mathrm{K}$-uptake by quinoa plants at flowering stage or K-uptake by straw at harvest stage. In addition, the values of $\mathrm{N}$-uptake at flowering stage and K-uptake by straw at maturity stage not influenced significantly by the interaction between $\mathrm{N}$-fertilizer rates and micronutrient fertilizer rates. On the other hand, data in Tables 4, 5, 7 and continuous reveal that the values of protein content, N, Puptake by straw and grains and K-uptake by grains as well as total $\mathrm{N}, \mathrm{P}$ and $\mathrm{K}$ uptake at maturity stage significantly affected by $(\mathrm{N}$ rate $\times$ bio-fertilizer rat) and ( $\mathrm{N}$ rate $\times$ micronutrient fertilizer rate). With respect to the effect of interaction between $\mathrm{N}$-fertilizer levels and micronutrient fertilizer rate, data explain that the interaction effect gave positive response on the studded chemical characters at the two growth stages except P-uptake by quinoa plant organs as well as total Puptake at maturity stage. 
Table 7. Interaction effect of nitrogen, bio and micronutrients fertilizers on nutrient uptake by quinoa organs at harvesting Stage.

\begin{tabular}{|c|c|c|c|c|c|c|c|c|c|c|c|}
\hline \multicolumn{3}{|c|}{ Treatment } & \multicolumn{3}{|c|}{ N-uptake } & \multicolumn{3}{|c|}{ P-uptake } & \multicolumn{3}{|c|}{ K-uptake } \\
\hline \multirow{2}{*}{$\begin{array}{c}\text { N- } \\
\text { Fert, } \\
\text { rate } \\
\left(\mathrm{kg}^{2} \mathrm{Fed}\right)\end{array}$} & \multirow{2}{*}{$\begin{array}{c}\text { Bio, } \\
\text { rate } \\
(\mathrm{L} \text { Fed }\end{array}$} & \multirow{2}{*}{$\begin{array}{l}\text { Micro, } \\
\text { rate } \\
\left.\text { (L Fed } d^{1}\right)\end{array}$} & \multicolumn{9}{|c|}{$\operatorname{gram} \mathrm{pot}^{-1}$} \\
\hline & & & Stratu & Graim & Total & Strax & Grain & Total & Strax & Graim & Total \\
\hline \multirow{6}{*}{$\mathrm{N}_{0}$} & \multirow{3}{*}{$b_{0}$} & $T_{0}$ & 0.202 & 0.192 & 0.394 & 0.009 & 0.024 & 0.033 & 0.443 & 0.172 & 0.615 \\
\hline & & $T_{1}$ & 0.211 & 0.210 & 0.421 & 0.010 & 0.026 & 0.036 & 0.433 & 0.183 & 0.671 \\
\hline & & Mesu & 0.205 & 0.201 & 0.407 & 0.009 & 0.025 & 0.034 & 0.453 & 0.190 & 0.643 \\
\hline & \multirow{3}{*}{$b_{1}$} & $\mathrm{~T}_{0}$ & 0.223 & 0.234 & 0.457 & 0.011 & 0.027 & 0.038 & 0.524 & 0.195 & 0.719 \\
\hline & & $T_{1}$ & 0.240 & 0.275 & 0,516 & 0.012 & 0.029 & 0.041 & 0.575 & 0.209 & 0.783 \\
\hline & & Mens & 0.232 & 0.255 & 0.456 & 0.011 & 0.028 & 0.039 & 0.549 & 0.202 & 0.751 \\
\hline \multirow{6}{*}{$\mathrm{N}_{1}$} & \multirow{3}{*}{$b_{b}$} & $\mathrm{~T}_{0}$ & 0.814 & 0.669 & 1.483 & 0.020 & 0.051 & 0.071 & 1026 & 0.438 & 1.454 \\
\hline & & $\mathrm{T}_{1}$ & 0.938 & 0.728 & 1.666 & 0.037 & 0.057 & 0.094 & 1.053 & 0.458 & 1551 \\
\hline & & Mesn & 0.876 & 0.699 & 1.574 & 0.03 & 0.054 & 0.083 & 1.044 & 0.463 & 1.507 \\
\hline & \multirow{3}{*}{$b_{1}$} & $\mathrm{~T}_{\square}$ & 0917 & 0.816 & 1.733 & 0.023 & 0.059 & 0.052 & 1.059 & 0.517 & 1.577 \\
\hline & & $\mathrm{T}_{1}$ & 0.951 & 1.017 & 1.968 & 0.026 & 0.065 & 0.091 & 1.028 & 0.547 & 1575 \\
\hline & & Mexn & 0.934 & 0917 & 1.851 & 0.024 & 0.052 & 0.057 & 1.044 & 0.532 & 1.576 \\
\hline \multirow{6}{*}{$\mathrm{N}_{2}$} & \multirow{3}{*}{$b_{0}$} & $\mathrm{~T}_{0}$ & 1050 & 0.790 & 1.870 & 0.027 & 0.060 & 0.087 & 1.159 & 0.462 & 1.621 \\
\hline & & $\mathrm{T}_{1}$ & 1.105 & 0.879 & 1994 & 0.029 & 0.073 & 0.102 & 1.037 & 0.492 & 1329 \\
\hline & & Mens & 1.092 & 0.835 & 1927 & 0.028 & 0.055 & 0.095 & 1098 & 0.477 & 1.575 \\
\hline & \multirow{3}{*}{$b_{1}$} & $\mathrm{~T}_{0}$ & 1.115 & 0.348 & 1963 & 0.030 & 0.066 & 0.097 & 1.094 & 0.518 & 1.612 \\
\hline & & $\mathrm{T}_{1}$ & 1.166 & 1.051 & 2217 & 0.035 & 0.072 & 0.107 & 1.346 & 0.531 & 1.877 \\
\hline & & Mesu & 1.140 & 0950 & 2.090 & 0.033 & 0.059 & 0.102 & 1.220 & 0.525 & 1.744 \\
\hline \multirow{6}{*}{$\mathrm{N}_{3}$} & \multirow{3}{*}{$b_{0}$} & $\mathrm{~T}_{0}$ & 0.724 & 0.607 & 1.331 & 0,026 & 0.040 & 0.066 & 1.010 & 0.310 & 1.321 \\
\hline & & $\mathrm{T}_{1}$ & 0.901 & 0.728 & 1.629 & 0.029 & 0.046 & 0.075 & 0.535 & 0.427 & 1.311 \\
\hline & & Mesi & 0.813 & 0.665 & 1.450 & 0.027 & 0.043 & 0.070 & 0.947 & 0.369 & 1316 \\
\hline & \multirow{3}{*}{$b_{1}$} & $\mathrm{~T}_{\square}$ & 0.535 & 0.657 & 1.372 & 0.021 & 0.043 & 0.064 & 0.969 & 0.298 & 1.268 \\
\hline & & $\mathrm{T}_{1}$ & 0.952 & 0.819 & 1.501 & 0.027 & 0.058 & 0.055 & 1.116 & 0.409 & 1525 \\
\hline & & Mesn & 0933 & 0.753 & 1.656 & 0.024 & 0.050 & 0.075 & 1.043 & 0.354 & 1396 \\
\hline \multirow{6}{*}{$\mathrm{N}_{4}$} & & $\mathrm{~T}_{0}$ & 0.978 & 0.725 & 1.703 & 0.034 & 0.062 & 0.096 & 1.035 & 0.445 & 1.450 \\
\hline & $b_{0}$ & $\mathrm{~T}_{1}$ & 1.028 & 09938 & 1.966 & 0.032 & 0.092 & 0.124 & 0.972 & 0.487 & 1.459 \\
\hline & & Mean & 1.003 & 0.832 & 1.835 & 0.033 & 0.077 & 0.110 & 1.003 & 0.456 & 1.470 \\
\hline & & $\mathrm{T}_{0}$ & 1.008 & 0.830 & 1.333 & 0.031 & 0.056 & 0.097 & 1.001 & 0.564 & 1565 \\
\hline & $b_{1}$ & $\mathrm{~T}_{1}$ & 1.021 & 1.039 & 2060 & 0.040 & 0.079 & 0.119 & 1.059 & 0.591 & 1.600 \\
\hline & & Menu & 1.014 & 0.935 & 1949 & 0.035 & 0.072 & 0.108 & 1.045 & 0.577 & 1.623 \\
\hline & & $\mathrm{T}_{0}$ & 0.762 & 0.586 & 1.348 & 0,022 & 0.047 & 0.069 & 0.859 & 0.381 & 1.239 \\
\hline & $b_{0}$ & $\mathrm{~T}_{1}$ & 0.693 & 0.649 & 1342 & 0.018 & 0.053 & 0.071 & 0.713 & 0.379 & 1.091 \\
\hline & & Mexn & 0.728 & 0.617 & 1.345 & 0.020 & 0.050 & 0.070 & 0.785 & 0,390 & 1.165 \\
\hline $\mathrm{N}_{s}$ & & $\mathrm{~T}_{0}$ & 0.748 & 0.677 & 1.425 & 0,025 & 0.045 & 0.070 & 0.731 & 0.313 & 1.045 \\
\hline & $b_{1}$ & $\mathrm{~T}_{1}$ & 0.795 & 0.766 & 1561 & 0.027 & 0.050 & 0.077 & 0.942 & 0.325 & 1.267 \\
\hline & & Mexin & 0.772 & 0.721 & 1.493 & 0.026 & 0.043 & 0.074 & 0.837 & 0.319 & 1.156 \\
\hline & & $\mathrm{T}_{0}$ & 0.307 & 0.330 & 0.637 & 0.018 & 0.044 & 0.062 & 0.443 & 0.223 & 0.666 \\
\hline & $b_{0}$ & $\mathrm{~T}_{1}$ & 0.287 & 0.436 & 0.743 & 0.022 & 0.051 & 0.073 & 0.494 & 0.267 & 0.760 \\
\hline$x$ & & Mesn & 0.297 & 0.393 & 0.690 & 0.020 & 0.047 & 0.057 & 0.459 & 0.245 & 0.713 \\
\hline$N_{6}$ & & $\mathrm{~T}_{0}$ & 0.296 & 0.512 & 0.508 & 0.016 & 0.048 & 0.064 & 0.602 & 0.228 & 0.831 \\
\hline & $b_{1}$ & $\mathrm{~T}_{1}$ & 0.308 & 0.568 & 0.875 & 0.019 & 0.052 & 0.072 & 0.629 & 0.244 & 0.573 \\
\hline & & Mesn & 0.302 & 0.540 & 0.842 & 0.018 & 0.050 & 0.068 & 0.516 & 0.235 & 0.852 \\
\hline
\end{tabular}




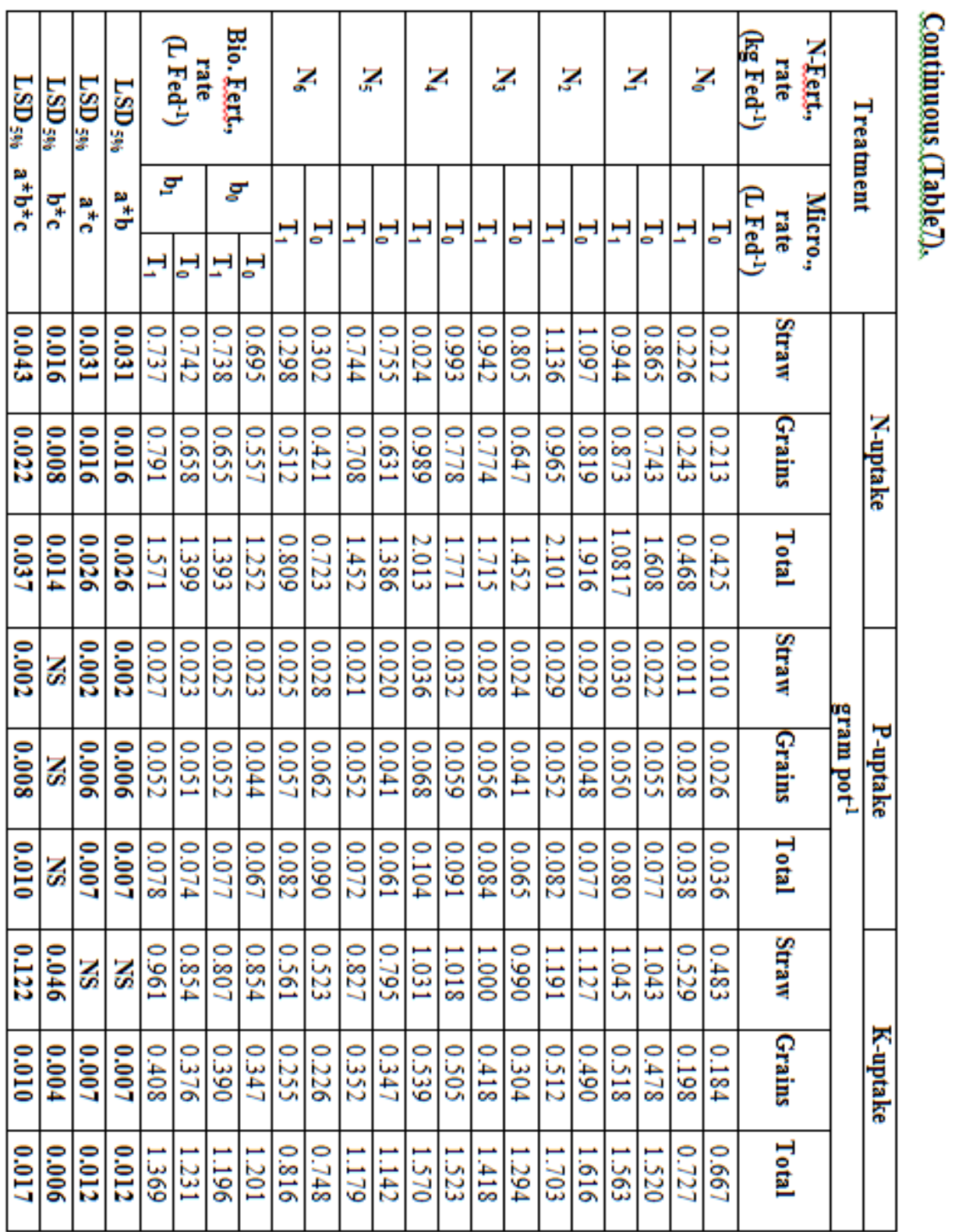


Generally, the statistical analysis of variance for data explicitly indicate that the values of all studded chemical characters at flowering and harvest stages markedly affected by $(\mathrm{N}$-fertilizer rates $\times$ bio-fertilizer rates $\times$ micronutrient fertilizer rates). The best interaction treatment that achieved the highest values for $\mathrm{N}, \mathrm{P}, \mathrm{K}$-uptake at flowering stage, protein content, $\mathrm{N}$-uptake by quinoa organs, $\mathrm{P}$ uptake by straw, K-uptake by straw as well as total K-uptake were $\left(\mathrm{N}_{2} \times \mathrm{b}_{1} \times \mathrm{T}_{1}\right)$. On the contrary, the highest values for P-uptake by grains and total $\mathrm{P}$ - uptake were recorded with treatment of $\left(\mathrm{N}_{4} \times \mathrm{b}_{0} \times \mathrm{T}_{1}\right)$. These results are in conformity with those obtained by Dhaliwal et al. (2019) and El-Gamal et. al. (2020) whom reported that combined application of organic , mineral and bio-fertilizers played a pivotal role in improvement of soil physico-chemical properties, macro and micronutrients distribution and their transformations, which leads to significant increasing of nutrients concentration and uptake by quinoa plants.

\section{Conclusion}

Quinoa as a grain crop has a high tolerance that enables it to grow in places where other crops cannot grow thus gives a better reaction and performance reflected on its growth, grain productivity and quality as well as organs chemical composition when it is amended with mineral, organic and bio fertilizers. Nonetheless, from a physiological activity perspective, the plant responded better to mineral fertilization. Even though the organic fertilizer did not contribute to the nitrogen available to the plant, the used bio and chelated micronutrients fertilizers are efficient in the contribution of nitrogen, phosphorus and potassium for plant development. The obtained results from this work indicate that application of nitrogen (mineral and organic), bio-and micronutrients fertilizers gave positive effect on most studied traits and the best treatment was the interaction treatment of $\mathrm{N}_{2}\left(100 \mathrm{~kg}\right.$ inorganic-N fed $\left.{ }^{-1}\right) \times \mathrm{b}_{1}\left(2 \mathrm{~L}_{\text {bio-fertilizers fed }}{ }^{-1}\right) \times \mathrm{T}_{1}\left(2 \mathrm{~L}_{\text {chelated }}\right.$ micronutrient fed $\left.^{-1}\right)$.

Finally, since the quinoa crop is a multi-purpose cereal crop with a high nutritional value and its cultivation succeeds in areas that suffer from problems in soil fertility, water stress, calcium carbonate stress and other problems that limit the productivity of other grain crops, the cultivated areas must be expanded with focusing on developing a balanced fertilization program based on organic and biological fertilizers in order to improve the physical, chemical and biological properties of these soils and thus increase the yield of quinoa to bridge the gap in the production and import of wheat from abroad, reduce the use of mineral fertilizers and their production costs and preserve the environment from pollution. 


\section{REFERENCES}

Abdulrahman M., Badran A. E. and Algosaibi A.M.(2019). Evaluation of efficiency and response of quinoa plant to nitrogen fertilization levels. Middle East J. Appl. Sci., Vol. 9(4): 839-849.

Ashik, J.M., Shamsuddoha, A. T. M and Nazmul Haque, Md. (2016). Effect of organic and inorganic fertilizer on the growth and yield of rice (Oryza sativa L.). Nature and Sci., Vol. 14(2):45-54.

Beigzade, M., Maleki, A., Siaddat, S.A. and Mohammadi.M.M. (2013). Effect of combined application of phosphate fertilizers and phosphate solubilizing bacteria on yield and yield components of maize single cross. Int. J. Agric. Crop Sci., Vol. 6 (17): 1179-1185.

Bender, R.R.; Haegele, J.W.; Ruffo, M.L.; Below, F.E.(2013). Nutrient uptake, partitioning, and remobilization in modern, transgenic insect-protected maize hybrids. Agron. J., Vol. 105: 161-170.

Bilal M., Muhammad, T., Irfan, A., Abdul, B., Bilal, A., Umair, K., Muhammad, Z., Naveed, A.(2017). Impact of integrated fertilization (organic and in-organic) on grain yield of maize. Agric., Forestry and Fisheries, Vol. 6 (5): 178-183.

Chukwu, L.I., Ano, A.O. and Asawalam, D.O. (2012). Effects of poultry manure and NPK fertilizer on soil properties and nutrient uptake of maize (Zea mays L.) plants growth in an ultisol. Proceedings of the 36th Annual Conference of the Soil Science Society of Nigeria (SSSN) on 7th - 11th March, 2012 University of Nigeria Nsukka.

Dhaliwal, S. S., Naresh, R. K., Agniva Mandal, M. K. W., Raj K. G., Rajveer S. and Dhaliwal, M. K. (2019). Effect of manures and fertilizers on soil physical properties, build-up of macro and micronutrients and uptake in soil under different cropping systems. A review, J. of Plant Nutri., Vol.42 (20): 2873-2900.

Eisa S., Hussin S., Geissler N., Koyro H.W. (2012). Effect of $\mathrm{NaCl}$ salinity on water relations, photosynthesis and chemical composition of quinoa (Chenopodium quinoa Willd.) as a potential cash crop halophyte. Australian J. of Crop Sci., Vol.6:357-368.

El-Gamal B.A., Hanan M. Abu El-Fotoh and Mervat A. H. (2020). Impact of organic and bio-fertilizers on soil health and production of quinoa and soybean. Middle East J. Agric. Res., Vol. 9 (4): 828-847.

Ewis,A.M.G. (2019). Evaluation the effect of $\mathrm{N}$ mineral fertilization in combination with $\mathrm{N}$ bio-fertilizer on barley yield and its components in sandy soil. J. Soil Sci. and Agric. Eng., Mansoura Univ., Vol. 10 (8):423-433. 
Ewis,A.M.G. (2020). Effect of P mineral fertilization combined with compost and phosphate solubilizing bacteria on wheat yield and its components in calcareous soil. J. of Soil Sci. and Agric. Eng., Mansoura Univ., Vol. 11 (10): 541-549.

Garcia M., Condori B., Del Castillo C. (2015). Agro ecological and agronomic cultural practices of quinoa in South America. In Quinoa: Improvement and Sustainable Production, pp. 25- 45. Eds K. Murphy and J. Mataguihan. New Jersey, USA: John Wiley \& Sons, Inc., EEUU.

Hakan, G. (2015). Effects of different nitrogen levels on the grain yield and some yield components of quinoa (chenopodium quinoa willd) under Mediterranean climatic conditions. Turk J. Field Crops, Vol. 20 (1): 59-64.

Heba M.A., K., Ragab, M.E., Youssef S.M. and Aisha H. A. (2019). Effect of different nitrogen fertilizer rates and sources on leaf chemical compositions and yield of quinoa plant as a new leafy vegetable crop. 14th Conf. Agric. Dev. Res., Fac. Agric., Ain Shams Univ., Cairo, Egypt., Vol. 27(1): 673 689.

Karina B. R., Stefania B., Rómulo O., and Ian S. A. R. (2014). Quinoa biodiversity and sustainability for food security under climate change. A review. Agron. Sustain. Dev., Vol. 34:349-359.

Lakudzala, D.D. (2013). Potassium response in some Malawi soils. International Letters of Chemistry, Physics and Astronomy, Vol.8 (2): 175-181.

Niguse, K., and Kassaye, M. (2018). Response of food barley (Hordeum vulgare L.) varieties to rates of nitrogen fertilizer in Limo District, Hadiya Zone, Southern Ethiopia. J. of Natural Sci. Res., Vol. 8 (15):17 - 31.

Oelke E.A., Putnam D.H., Teynor T.M. and Oplinger E.S. (1990). Quinoa, in alternative field crops manual, University of Wisconsin - extension, cooperative extension, Univ. of Minnesota - Center for Alternative Plant and Animal Products, Minnesota Extension Service.

Parra M. G, Molano G. J, and Oyola Y.D. (2019). Physiological performance of quinoa (Chenopodium quinoa Willd.) under agricultural climatic conditions in Boyaca, Colombia. Agron. Colomb. Vol. 37 (2): 144-152.

Place, F., Barrett, C. B., Freeman, H. A., Ramisch, J. J. \& Vanlauwe, B. (2003). Prospects for integrated soil fertility management using organic and inorganic inputs: evidence from smallholder African agricultural systems. Food Policy, Vol.28 (4): 365-378.

Rehm G. and M. Schmitt. (2002). Potassium for crop production .Univ. of Minnesota Extension. (http://www.extension.umn.edu/distribution/crop systems/DC6794.html). 
Ronaled, E.W., Acree, T.E., Deckar, E.A., Penner, M.H., Reid, D.S., Schwartz, S.J., Shoemaker, C. F., Smith, D. and Sporns, P. (2005). Hand Book of Food Analytical Chemistry pub. By Wiley, J. and Sons, Inc., Hoboken, New Jersey Pub. Simultaneously in Canada.

Sarwar G., H., Hussain S. N., S., Muhammad, M. I. and Safdar E. (2008). Improvement of soil physical and chemical properties with compost application in rice wheat growing system. Pak. J. Bot., Vol.40 (1): 275-282.

Shafi, M., Bakht, J., Jalal, F., Khan, M. A. and Khattak, S. G. (2011). Effect of nitrogen application on yield and yield components of barley (Hordeum vulgare L.). Pak. J. Bot., Vol. 43(3): 1471-1475.

Singh G., Sarvanan S., Rajawat K. S., Rathore J.S. and Singh G. (2017). Effect of different micronutrients on plant growth, yield and flower bud quality of broccoli (Brassica Oleracea Var. Italica). Curr. Agri. Res., Vol. 5(1): 108-115.

Somani LL. (2008). Micronutrients for soil and plant health. Agrotech Publishing Academy. 14 -74. Website: www .agrotechbooks.com.

Sparks, D.L. (1996). "Methods of Soil Analysis", (Ed.) Part 3-chemical methods, (2nd Ed.), Agron. 9: A.S.A., Ins., Madison, Wisc., USA.

Stewart Z. P., Paparozzi E. L., Wortmann C. S., Jha P. K. and Shapiro C.A. (2021). Effect of foliar micronutrients ( $\mathrm{B}, \mathrm{Mn}, \mathrm{Fe}, \mathrm{Zn})$ on maize grain yield, micronutrient recovery, uptake, and partitioning. https:// doi. org/ 10.3390/ plants 10030528

Taiwo LB, Adediran JA, Akande MO,Banjoko VA, Oluwatosin GA.(2001). Influence of legume fallow on soil properties and yield of maize in south western. Nigerian J. Agric. Trop. \& Subtrop., Vol. 102:109-117.

Umar Khan. M, Qasim M. and Ullah Khan I. (2007). Effect of integrated nutrient management of crop yields in rice-wheat cropping system. Sarhad J. Agric., Vol. 23 (4): 109-1026.

Vessey, J.K. (2003). Plant growth promoting rhizobacteria as biofertilizers. Plant Soil, Vol.255:571-586.

Wali1, A. M., Shamseldin, A., Radwan, F. I., Abd El Lateef, E. M. and Zaki, N. M.(2018). Response of barley (Hordeum vulgare) cultivars to humic acid, mineral and biofertilization under calcareous soil conditions. Middle East J. Agric. Res., Vol.7(1): 71-82.

Westerman (Ed.) (1990). Soil Testing and Plant Analysis, (3rd Ed.), Monograph No.3, Soc., of Am. Book Series, S.S.A., Ins., Madison, Wisc., USA. 


\section{تأثر محصول الكينوا ومكوناته بالتسميد النيتروجيني والأسمدة الحيوية والمغذيات الاقيقة في التربة الجيرية}

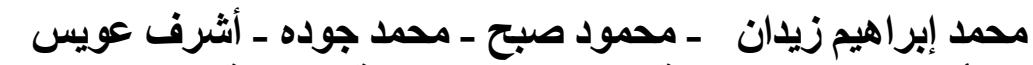
قسم الأراضي والمياه ـ كلية التكنولوجيا والتنمية ـ جامعة الزقازيق ـ ـ مصر ـ

أجريت تجربة أصص بكلية التكنولوجيا والتتمية بجامعة الزقازيق بمحافظة الثرقية بمصر التمبر

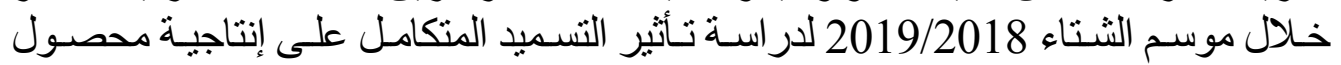

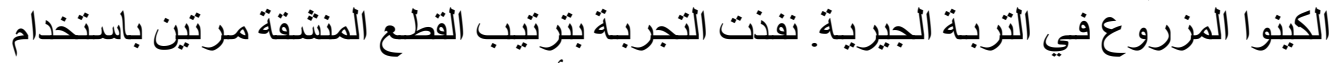

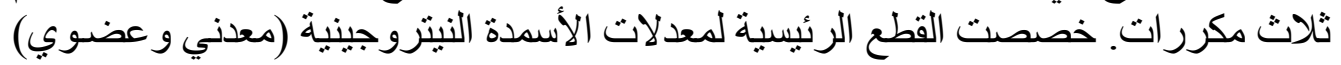
وهي:

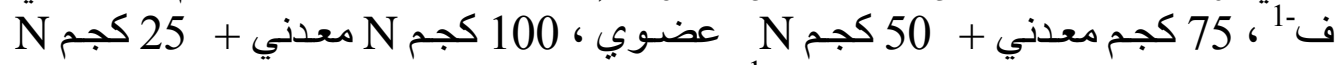

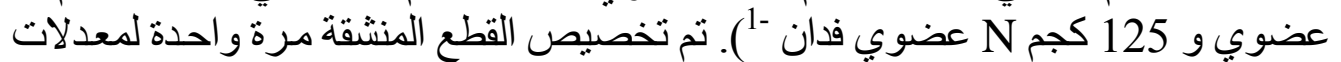

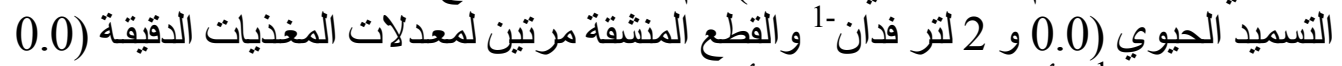

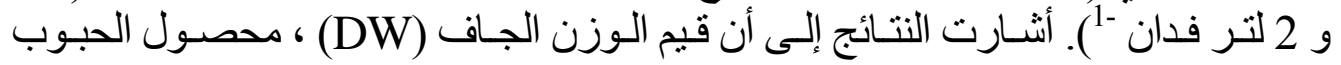

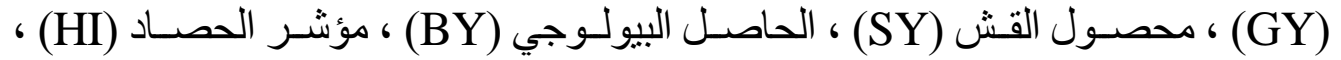

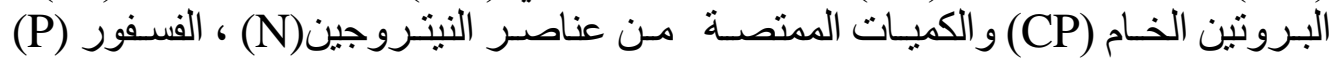

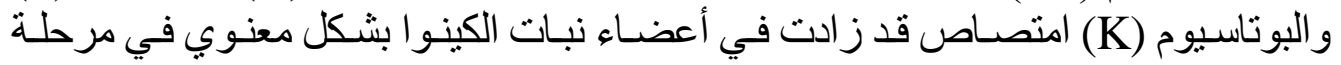

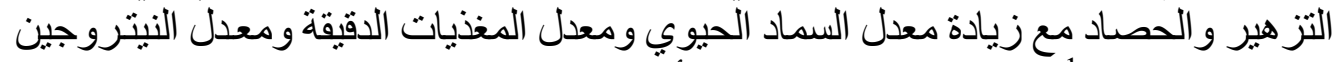

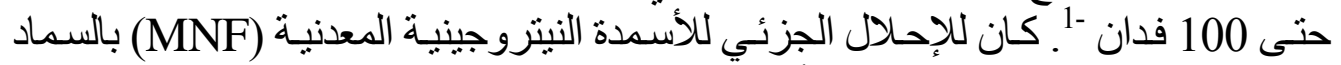

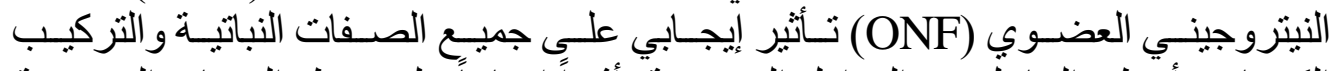

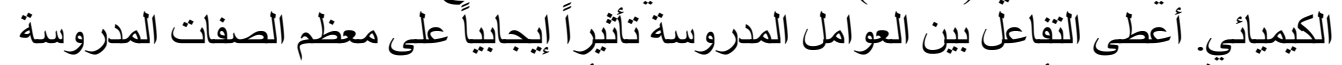

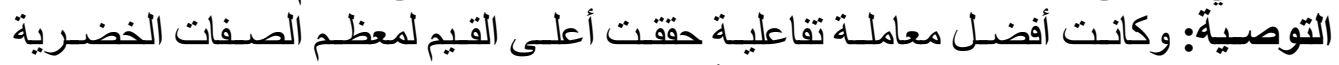
و الصفات الكيميائية هي $)\left(T_{1} \times b_{1} \times N_{2}\right.$ أو $\left(T_{1} \times b_{1} \times N_{4}\right)$. 